\title{
Convolutional Neural Network Based Path Navigation of a Differential Drive Robot in an Indoor Environment
}

\author{
Prithvi Krishna C, Vasanth Kumar CH
}

\begin{abstract}
The current work illustrates a vision-guided approach to a real-time robot navigation system and the implementation of Faster Convolutional Neural Networks (FCNN) to train and detect objects with multiple datasets of the mobile robot and obstacles. The algorithm keeps monitoring the distance between the obstacles and generates way points inbetween the obstacles in such a way that a path is created towards the target. Thus, the shortest path for navigation is created which checks for possible errors and update the path during execution, making it an AI system. This approach reduces the need for incorporating multiple EMU sensors on the mobile robot and transfers the computation process to a remote processor. The processor and mobile robot communicate wirelessly for simultaneous localization and path planning. While the algorithm is being executed, trained objects are detected from each frame captured by the camera which is used to develop path by avoiding the obstacles. The performance of the system is evaluated by conducting multiple experiments with different mapping regions.
\end{abstract}

Keywords - Robot Navigation, Path Planning, Collision Avoidance, Object Recognition, Mapping Techniques, FCNN.

\section{INTRODUCTION}

Path designing is a crucial advancement in mobile robot navigation. Path planning refers to how the mobile robot searches for an optimal path from initial point to the target point in an unknown environment. The performance is based on the parameters like angular velocity, linear velocity and wheel diameter. The artificial potential method is widely used because of its fast response speed, small computation amount, convenient actual time control and better path generation. But this methodology is typically utilized in static condition. But in Realtime applications, the mobile robot condition isn't stationary, the goal and objects are dynamic.

Generally, path planning uses vision to identify the position of bot with respect to the goal point. [1] in 2014 proposed "An Image Based Path Planning and Motion Planning for Autonomous Robot" in which path for robot is planned based on image processing techniques. First, the environment is captured using a camera. Then, the image is converted to gray scale image to identify the obstacles using two fundamental image processing techniques like edge extraction and thresholding where it gets the outline of the obstacle to avoid.

Prithvi Krishna C, Mechanical Engineering SRM IST Chennai, T.N India. (E-mail: prithvikrishna1911@gmail.com)

Vasanth Kumar CH, Mechanical Engineering SRM IST Chennai, T.N, India. (E-mail: sreevasanthkumarchoda@gmail.com)
Revised Manuscript Received on July 10, 2019.

Then $A^{*}$ star search i.e., best first search is performed to reach the goal point in shortest distance. [2] in 2017 proposed "Development of Robot Navigation Method Based on Single Camera Vision Using Deep Learning" which utilizes vision for robot navigation on roads for real-time approach. During navigation, a camera captures the image of the environment and then the image is transformed into an illumination variant picture. After that, Deep Belief Neural Network (DBNN) takes the picture as a major input. It grabs the road characteristics layer-by-layer for detection. It follows Integrating, understanding the surroundings, localization, and path planning using only one camera. In this paper path planning is done using live streaming of video to continuously monitor the position of bot for better obstacle avoidance.

Machine learning permits computational systems that are made from numerous processing layers to learn representations of data with various dimensions of reflection. [3] in 2018 proposed "Object Detection Based on Deep Learning of Small Samples". It represents an object identifier based on machine learning of little examples. The algorithm can train samples automatically by generating synthetic samples to solve the issue of few examples. Then, machine learning and high prediction systems are utilized in the Deep Convolutional Neural Systems. The semantic relevance of objects is used to increase the precision of feature detection in complex situations. [4] in 2016 proposed You Only Look Once, is an advanced way to deal with object identification. Earlier work on object recognition reimposes classifiers to perform identification. Here, object detection works with an algorithm to spatially differentiate bounding boxes and corresponding class probabilities. A single neural network predicts bounding boxes and class probabilities directly from full images in one evaluation. Since the entire detection works is a single network, it can be optimized end-to-end specifically on recognition execution. This unified system is amazingly quick with a frame rate of 45 frames per second.

The novelty with this paper is to put together all these concepts and train the robot to reach the goal position in shortest path with no collisions and less turnings in the shortest time possible. In this paper, the overall system's work process is divided into four major segments. Section 2 gives an overview of The Robot Path Planning using Vision. Section 3 describes about Robot Identification and Obstacle

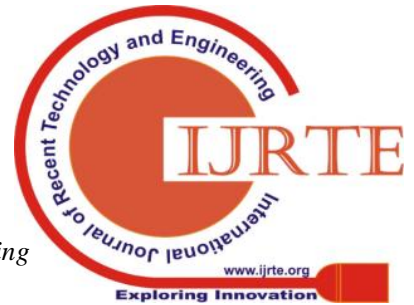




\section{CONVOLUTIONAL NEURAL NETWORK BASED PATH NAVIGATION OF A DIFFERENTIAL DRIVE ROBOT IN AN INDOOR ENVIRONMENT}

Detection. In Section 4, Working and Monitoring Waypoints. Finally, this paper finishes with section 5, by concluding and describing the scope of future extensions, followed by references.

\section{ROBOT PATH PLANNING USING VISION}

In this section, the issue of path planning utilizing vision with no collision for a Bot with two degrees of movement is studied. By using vision, the Bot should choose a way that imitates an easily accessible and nearer to the way a human would take in a similar type of environment to achieve the goal point from the initial point. This basically implies the accompanying: (a) The way must be short , that is, it must not contain longer deviation when altogether shorter path is possible; (b) It must have an ensured measure of clearance, that is, the separation of any point on the way to the nearest impediment must not be lower than some specified value; and (c) Turning must be smooth, that is, path should not contain any sharp turns.

The Visibility Graph (VG) is the mostly used structure for figuring the collision less and straightaway path from start to goal point. The path of visibility graph will be a straight-line path from start point to goal point. In visibility graph, start point, goal point and obstacles are identified. Then, shortest path from the initial point to end point is taken in such a way that it must pass through edges of the obstacles while maintaining a prescribed distance between the edge of the obstacle and the bot, so that, it does not hit any obstacle. All obstacle vertices in visibility graph, initial and goal point positions are considered. A* search is utilized to identify shortest path from initial point to goal point, passing through vertices of the obstacles. The key advantage is that, it depends only on number of obstacles, and the major disadvantages are that the robot path is very close to obstacles and exact positions of the obstacles is not defined.

On the other hand, Cell decomposition is the method utilized for path planning where the entire workspace is divided into different segments. Each segment is divided based on the vertices of the obstacle. The Bot should follow the shortest path where passing through the segments with no vertices and avoiding segments with vertices. Even this method has sharp turns and follows longest path, which is not suitable for this paper.

Then again, path planning utilizing the Voronoi diagram for the obstructions yields a way with more clearance, Initially, consider the vertices of the polygonal objects with polygon into little sections. Next, finding the Voronoi graph for the path from initial point to goal point. When this convoluted Voronoi path is built, the distance is maintained between the bot and the object by making a boundary around it. The bot should follow the path, passing outside those boundaries and reach the goal point. Path achieved in Voronoi diagram is relatively not the shortest path when compared with the visibility graph, but it follows completely collision free path. Voronoi diagram follows Dijkstra's algorithm to search the shortest path from initial point to goal point without hitting obstacles with no sharp turns. A part of this method is implemented in this paper for path planning of the bot from initial point to reach target point with shortest path, no collisions and minimal turns. a huge number of positions and subdividing each of the

\section{ROBOT IDENTIFICATION AND OBSTACLE DETECTION}

Normally, for Bot feature extraction, camera is set for a settled region of interests in a specific video arrangement. Setting Region of Interest (ROI) at a few points of interest, for example, constraining the bot pictures to an appropriate size range. Additionally, by setting appropriate areas, the required zone will be diminished, and the principle features of robot can be ensured to be captured by the camera. At that point, a pre-trained Convolutional Neural Network can yield precise robot features to identify robot in the chosen area and create a bounding box around it.

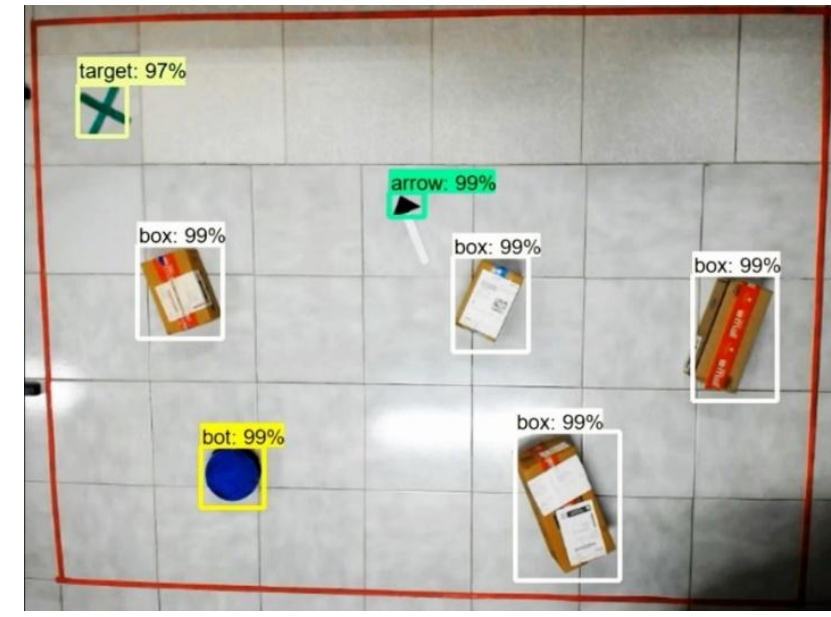

Fig. 1. Detecting Trained Objects

In Neural Networks, an image is received, remodelled and passed through sequence of hidden layers. All layers in the neural networks comprises of sets of neurons. Each and every neuron is completely associated with other neurons in the past layer. When an input is given, it holds raw pixel value. Then, it is sent through convolutional layer where it performs dot product between their weights followed by Pooling operation where downsizing the spatial dimensions of the image is performed. Finally, the class score of the image is obtained.

Initially, the system needs to be trained with adequate images of each object. The dataset consists of 200 images of each set of customized bot, obstacles and target. During the train session, the system performs mathematical operations on the images and applies a filtering-mask to identify the common features in all the images of that particular class of dataset. A graph is plotted continuously during the training session to determine the Loss Function. The training operation is performed until the loss graph is linear. It also generates a model with set of scores for the input images.

While performing deep learning of the image, it has two datasets- Training data and Validating data. For every step of training, validation loss gets lower and accuracy gets higher. Also, the system needs to identify the trained objects from the video frame. While testing, the algorithm extracts key features from the live feed, by comparing the live feed data with the model file to extract pretrained objects from the frame. If the final score of the image matches with 
trained data, it gives true or else false. The probability of matching depends on the dataset provided or accuracy of the system. Higher the number of training steps, greater will be the detection accuracy. But, by increasing the number of detection objects will significantly reduce the accuracy.

\section{WORKING AND MONITORING WAY-POINTS \& RESULTS}

A camera is placed at the top of the wall mount to capture the workspace of the mobile robot. The robot and processor are connected wirelessly. The Bot consists of wireless data transmission module, Motor driver, Micro geared motors, microcontroller and power source. The wireless module of the processor is used to send commands to the mobile robot for turning operations, such as Left, Right, Forward and Backward. These commands are received by the microcontroller and controlling signals are sent to motor driver. The micro geared motors are controlled by a corresponding driver channel.

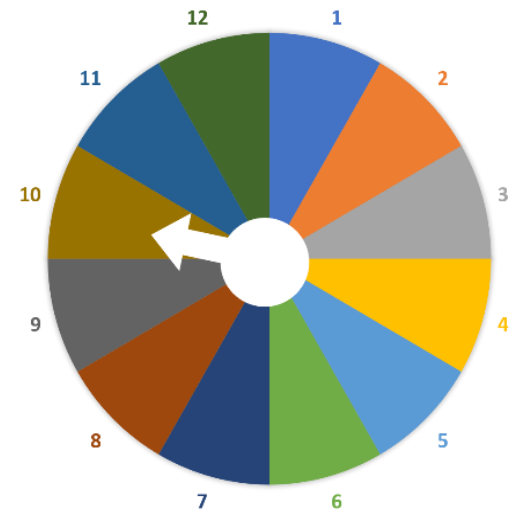

Fig. 2. Segmentation for Localization of Target

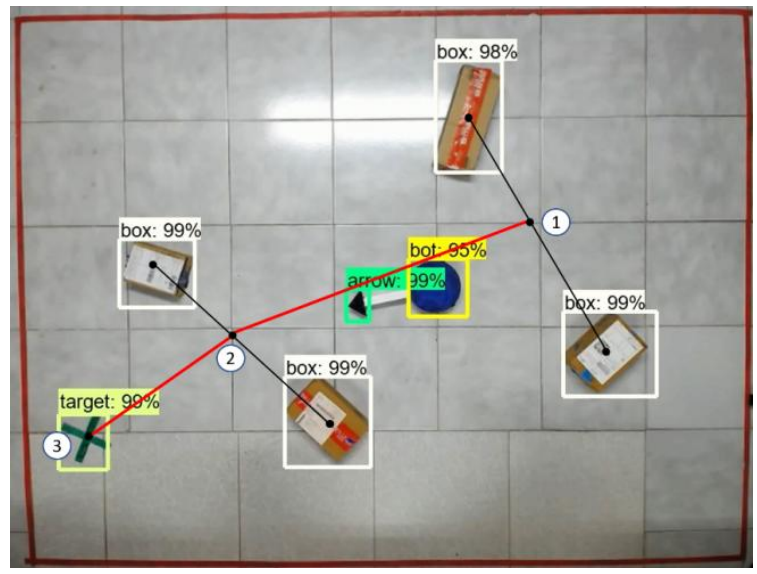

Fig. 3. Implementation of Path Planning

In the program, the area around the robot is divided into 12 sections in such a way that, robot is at the center of a big circle with sections of 30 degrees each labelled 1, 2, 3, 4 and so on till 12 , in clockwise direction. The algorithm detects the predefined objects from the video feed and gives $x-y$ coordinates of the objects, target and Bot. The algorithm then generates waypoints in the workspace which are at the the target. The robot locates the section in which the first waypoint is located and performs left or right operation until it reaches the same section as of the waypoint. The robot is maximum distance from the objects and in the direction of

then commanded to move forward until it reaches the first waypoint. This process is repeated for the subsequent waypoints till the target is reached.

\section{CONCLUSION}

In this paper, Fast-RCNN model is used to detect objects and define xy-coordinates for position estimation. The accuracy of detection of objects is based on the number of trained objects. If the number of trained objects increases, the detection rate reduces, thus the accuracy of detection reduces. The algorithm then generates path based on the xycoordinates obtained to be followed by the robot to reach target location by utilizing both position and velocity information. The use of sensors on the mobile robot reduces while transferring the computation to the remote processor. A demonstration was conducted in which, the mobile robot was capable of avoiding detected obstacles effectively.

As further study, research work is expected for making the system to be able to work in an outdoor environment The future scope of this project can be extended to home automation and outdoor navigation. This project can be used in industries for carrying heavy weights from point $A$ to point $\mathrm{B}$ by avoiding obstacles and detecting dynamic targets.

\section{REFERENCES}

1. H. N. Joshi and P. J. P. Shinde, "An Image Based Path Planning And Motion Planning for Autonomous Robot,", International Journal of Computer Science and Information Technologies, vol. 5, no. 4, pp. 4844-4847, 2014

2. K. M. I. Khalilullah, S. Ota, T. Yasuda, and M. Jindai, "Development of Robot Navigation Method Based on Single Camera Vision Using Deep Learning,", Proceedings of the SICE Annual Conference, pp. 939-942, 2017.

3. Ce Li, Yachao Zhang and Yanyun Qu, "Object Detection Based on Deep Learning of Small Samples,", Tenth International Conference on Advance Computational Intelligence, pp. 449-454, 2018.

4. J. Redmon, R. Girshick, A. Farhadi, and A. Dataset, "You Only Look Once : Unified, Real-Time Object Detection."

5. S. Gaijar, "Complete Coverage Path Planning Algorithm For Known 2d Environment,", IEEE International Conference on Recent Trends in Electronics Information \& Communication Technology, pp. 963-967, 2017.

6. Q. Han, "Path Planning of Mobile Robot Based on Improved Ant Colony Algorithm," 2011 Int. Conf. Consum. Electron. Commun. Networks, no. 1, pp. 531-533, 2011.

7. L. Yu, X. Chen, and S. Zhou, "Research of Image Main Objects Detection Algorithm Based on Deep Learning," 2018 IEEE 3rd Int. Conf. Image, Vis. Comput., pp. 70-75, 2018.

8. Sanjanna, Y. Bevish Jinila (2015), "An Approach on Automated Rescue System with Intelligent Traffic Lights for Emergency Services", International Conference on Innovations in Information, Embedded and Communication Systems (ICIIECS 2015), pp. 1-5, IEEE

9. Anu, V. Maria, D. Sarikha, G. Sai Keerthy, and J. Jabez. "An RFID based system for bus location tracking and display." In International Confernce on Innovation Information in Computing Technologies, pp. 1-6. IEEE, 2015.

10. Jeba Blessingson, Y.Bevish Jinila (2010), " Multi Utility Tracing Kit for vehicles using RFID Technology", Recent Advances in Space Technology Services and Climate Change -2010, PP. 273 - 276, IEEE 